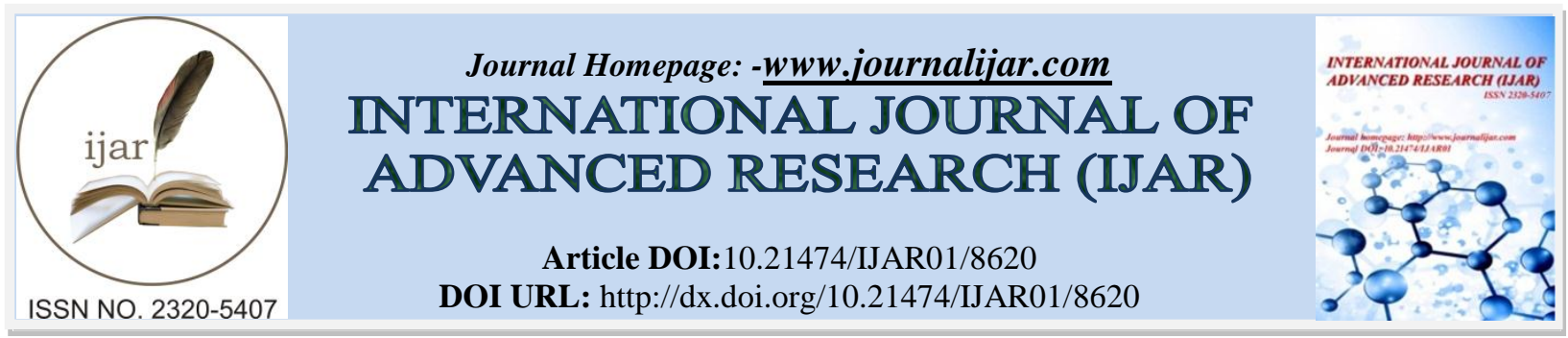

RESEARCH ARTICLE

\title{
INTRAORAL MOLAR DISTALIZATION APPLIANCES: A REVIEW.
}

\author{
Apurva Nikte ${ }^{1}$, Urvashi Nikte $^{2}$,Sushrut Niphadkar ${ }^{3}$, Vaidehi Arekar $^{4}$, Paritrat Prakash $^{5}$ and Utsav Nikte ${ }^{6}$ \\ 1. MDS Orthodontics and Dentofacial Orthopaedics, Private Practice, Mumbai. \\ 2. MDS Oral Medicine and Radiology, Private Practice, Mumbai. \\ 3. MDS Endodontics and Conservative Dentistry, Private Practice, Nashik. \\ 4. MDS Orthodontics and Dentofacial Orthopaedics, Private Practice, Mumbai. \\ 5. BDS, Private Practice Mumbai \\ 6. BDS, Private Practice Mumbai
}

\section{Manuscript Info}

\section{Manuscript History}

Received: 05 January 2018

Final Accepted: 07 February 2019

Published: March 2019

Key words:-

Non-extraction, Class II molar relation, Molar distalization.

\begin{abstract}
Recently there has been an increase in demand toward various conservative treatment methods to avoid extraction of healthy teeth and to increase patient compliance. Eliminating the need of extraction involves the correction of malocclusion which will result in improvement in facial profile, aesthetics and smile without loss of permanent tooth/teeth. In cases with minimal arch length discrepancy and angles class II molar relationship with orthognathic mandible and maxilla, distalization of molars creates additional space within the arch. Many appliances for class II correction need patient compliance, for example, headgears, elastics, etc. which may delay treatment results if the patient does not follow the instructions. Therefore there is a need for appliances which reduces patient compliance and gives effective results. This article reviews various intraoral appliances and methods for molar distalization.
\end{abstract}

Copy Right, IJAR, 2019,. All rights reserved.

\section{Introduction:-}

Current orthodontic treatment concepts have been directed towards conservative approaches to reduced the need for extraction. Class II malocclusions is one of the most common orthodontic cases which can be resolved by several methods. Correction of Class II malocclusion by non extraction approach can be achieved by both intraoral and extraoral forces. Molar Distalization method is one of the methods to correct Class II malocclusion which involves, distalization of molars by using extraoral or intraoral forces which causes increase in the arch length, in cases where maxilla and mandible is orthognathic. This method requires patient compliance when treated with headgear or elastics. Recently several intraoral appliances have been introduced to reduced patients compliance and maximize the efficiency of the results.

\section{Indications}

1. Class-II molar relationship due to the maxillary dentoalveolar protrusion.

2. End on molar relationship with mild to moderate space requirement.

3. Class I malocclusion canine with highly placed or impacted canine.

4. Lack of space for eruption of premolars due to mesial migration of permanent first molars 
5. Good soft tissue profile

6. Borderline cases

7. Mild to moderate space discrepancy with missing $3 \mathrm{rd}$ molars/2nd molars not yet erupted

8. Normal or hypo divergent growth pattern

9. Class-II subdivision cases requiring unilateral distal molar movement

10. Anchorage loss during active orthodontic treatment.

\section{Contraindications}

1. Retrognathic profile

2. Vertical growth pattern

3. $3^{\text {rd }}$ molars have erupted or close to eruption

4. Skeletal or Dental open bite

5. Patients with severe arch length tooth size discrepancy.

\section{Intraoral Appliances For Molar Distalization} Atkinson Buccal Bar:

Guerrero James in 1959 designed this device for moving posterior teeth distally. When minimum forces i.e., 2 ounces is applied this appliance moves the buccal segments posterioroly, with or without the presence of second molars. Anterior teeth were positioned using cervical anchorage.[1]

\section{Herbst Appliance:}

Emil Herbst in 1905, originally designed the appliance and was later popularised by Pancharz[2]. This appliance controls the direction of mandibular growth and also has the ability to inhibit maxillary growth in the saggital direction. The dental changes include distalization of the maxillary molars and mesial movement of the mandibular molars and incisors. In 1989, John R Valant[3] used the same appliance and observed 10mm of increase in the arch length. On cephalometric analysis he found that bodily molar distal movement of the molars had taken place.

\section{Jasper Jumper:}

In 1987, James J. Jasper made an appliance which was identical to the herbst device. The appliance comprises of two vinyl coated auxillary springs attached to banded upper and lower fixed appliances. In the posterior region, the springs are fixed to maxillary first molars and anteriorly to mandibular archwire. Transpalatal bar and lower lingual arch are used for anchorage. This appliance is similar to Herbst appliance it controls the direction of mandibular growth and also has the ability to inhibit maxillary growth in the saggital direction. This appliance also causes molar distalization and mesial movement of mandibular dentoalveolar segment.[4]

\section{Pendulum Appliance:}

In 1992, Higlers introduced this appliance which comprised of a Nance acrylic button for anchorage, 0.032" TMA swinging springs which transfers force to maxillary molars and a lingual sheath of 0.036 " in which the TMA spring is inserted. Activation of this device is done extraorally, the spring should be parallel to midsaggital plane when the device is place inside the mouth. The force applied on each side is nearly 200-250gms which creates a movement of 5mm within 3-4 months of period.[5]

\section{Modified Pendulum Appliance:}

In 1999, this modified Pendulum M was introduced by Scuzzo and he claimed that this appliance ensures bodily movement of molars. To achieve bodily movement of maxillary molar he invented the horizontal pendulum loop. Activation of the appliance is done by opening the loop because of buccal and/or distal movement of roots of molar.[6]

\section{Bone Anchored Pendulum Appliance:}

In 2006, Kirecelli C, Pekpas Z O, Kircelli BH developed this appliance which helps in molar distalisation without loss of anchorage. Palatal titanium implants are placed $7-8 \mathrm{~mm}$ distal to incisive foramen and 3-4 $\mathrm{mm}$ lateral to midline.[7]

\section{Molar distalization with Repelling Magnets:}

In 1988, Gianelly used repelling magnets to distalize maxillary molars. The magnets are inserted in the headgear tube of first maxillary molars and 0.014" ligature wire is passed through it. The magnets exerts a force of 200-225g 
but drops gradually as the space opens over $1 \mathrm{~mm}$. Anchorage can be reinforced by placing modified Nance appliance.[8]

\section{Franzulum appliance:}

It was introduced by Friedrich Byloff in 2000 for mandibular molar distalization. Anterior anchorage is reinforced using acrylic button of $5 \mathrm{~mm}$ width which also contains a tube. Rests on canine and premolars are placed using 0.032 " wire. NiTi coil springs are inserted in the tube which is present in the acrylic button. These springs exert 100$200 \mathrm{~g}$ of force on each side.[9]

\section{Jones Jig:}

It was developed by Jones and White in 1992, the appliance consists of a modified Nance device attached to the first premolar or deciduous second molar using heavy round wire and a light wire passing throught the molar tube, both the wires are joined to a fixed attachment sheath and hook. the open coil spring exerts $70-75 \mathrm{gms}$ of force which causes molar distalization of $2.5-2.8 \mathrm{~mm}$. [10]

\section{Saif Spring:}

Armstrong in 1970, introduced the pace spring which was later called as Saif spring. It is nickel-titanium closed spring that applies intermaxillary traction. It contains two springs within each other and loops fused to springs in both sides. It is attainable in $7 \mathrm{~mm}$ and $10 \mathrm{~mm}$ diameter.[11]

\section{K-loop Molar Distalizer:}

This appliance was introduced by Varun Karla in 1995. This appliance is fabricated using 0.017"x0.025" TMA wire where the loop is $8 \mathrm{~mm}$ in length and $1.5 \mathrm{~mm}$ in width. A $20^{\circ}$ bend is given in both mesial and distal legs. The mesial leg is marked $1 \mathrm{~mm}$ mesial to the mesial leg of the premolar bracket and the distal leg is marked $1 \mathrm{~mm}$ distal to the molar tube. The $20^{\circ}$ bends in appliance legs is reinforced by the activation movements as the loop is squeezed into place and this counteracts the force produced by the tipping movements of the appliance. Thus the distal movement of the molar occurs bodily rather than tipping. Reactivation of the appliance by $2 \mathrm{~mm}$ should be done after every $6-8$ weeks.[12]

\section{Forsus Fatigue Resistant Device:}

It is an interarch push spring device that produces force when fully compressed. This appliance has an intrusive effect on maxillary first molars. The distal end of the push rod passes into the telescopic cylinder and the hook on the mesial end is attached the canine and premolar region in the mandibular arch. The cylinder consists of inner and outer slide tubes which is surrounded by an open coil spring. The distal end of the cylinder is connected to the maxillary molar headgear tube with an $\mathrm{L}$ pin. The push rod compresses the spring when the patient closes his mouth. Thus this force is then transferred to the maxillary molars, using the mandibular arch as anchorage unit.[13,14]

\section{Fixed Piston Appliance:}

This device was developed by Green field in 1995. Fixed piston appliances causes translation of the maxillary first molars and requires no patient compliance. The appliance consists of bands on maxillary first molars and first premolars, 0.036 " stainless steel tubing which is soldered to the premolars, 0.030 " stainless steel wires soldered to the first molar bands, Nance button which is larger than standard size, $0.055^{\prime \prime}$ superelastic nickel titanium open coil spring. [15]

\section{Distal Jet Appliance:}

Carano and Testa introduced this appliance in 1996. It contains a Nance button which is attached with bilateral tubes in 0.036" diameter. Bayonet bends are given at the end of the wire which comes out of acrylic and is passed into palatal sheath on molar band. Wire is attached to NiTi coil spring with the help of screw clamp. Anchorage is provided with a wire which is soldered on the bands of first and second premolars from Nance palatal button.[16]

\section{Skeletalized Distal Jet Appliance:}

This appliance is similar to the Distal Jet appliance but instead of Nance palatal button it consists of palatal implant to provide absolute anchorage. this appliance also causes bodily movement of molars.[17] 


\section{Super elastic Nickel Titanium Wire:}

Locatelli invented this technique using super elastic nickel titanium wire with shape memory (NeoSentalloy) to move maxillary molars distally. Neo Sentalloy wire with regular archform is placed over the maxillary arch. Stops are crimped and hooks are added according to the markings made on the wire. Wire is inserted such that posterior stop abuts mesial end of molar tube, anterior stop abuts distal of premolar. Anchorage is reinforced by Nance appliance.[18]

\section{Skeletal Anchorage system:}

The skeletal anchorage system (SAS) consists of titanium anchor plates and monocortical screws that are temporarily placed in either the maxilla or the mandible, or in both, as absolute orthodontic anchorage units, Distalization of the molars has been one of the most difficult biomechanical problems in traditional orthodontics, particularly in adults and in the mandible, However, it has now become possible to move molars distally with the SAS to correct anterior crossbites, maxillary dental protrusion, crowding, dental asymmetries without having to extract premolars. Skeletal anchorage system (SAS) uses pure titanium anchor plates and screws as absolute orthodontic anchorage units. The anchor plates are monocortically placed at the piriform opening rim, the zygomatic buttresses, and any regions of the mandibular cortical bone, Because the anchor plates work as the onplant and the screws function as the implant, SAS enables the rigid anchorage that results from the osseointegration effects in both the anchor plates and screws.[19, 20]

The SAS has outstanding advantages not provided by the other mechanisms for distalizing the mandibular molars.

1. It is possible to intrude the mandibular molars with the SAS. Therefore the extrusion of the mandibular molars after the tipping of the molar distalization can be corrected easily.

2. En masse distalization of the mandibular buccal segments or the entire dentition is also possible if the mandibular dentition is aligned.

3. With the SAS, it is not always necessary to extract the mandibular first or second premolars even in patients with moderate to severe crowding.

4. Molar relationship in patients with symmetric or asymmetric Class III molar relationship can be corrected without having to extract mandibular premolars.

\section{Conclusion:-}

Since 1899 Edward H. Angle published the first article of classification of malocclusion, the position of the molars and the relationship between opposing molars has been an important topic of the orthodontic literature. Molar Distalisation has been an important treatment modality since there has been increased demand for nonextraction or conservative treatment options. Since there are various appliances available, every orthodontist should select an appliance according to the precise diagnosis which includes the age, growth pattern and basic concern of the patient. Anchorage loss is a major concern of intraoral molar distalizers, but since the introduction of molar distalizers with miniimplants this problem can be avoided.

\section{References:-}

1. Guerrero James J. Posterior movement of the buccal segments. Am J Orthod.1959;45(2):125-30.

2. Pancherz H. Treatment of Class II malocclusion by jumping the bite with the Herbst appliance. A cephalometric investigation. Am J Orthod. 1979;76(2):423-42.

3. Valant JR. Increasing maxillary arch length with modified Herbst appliance. J Clin Orthod. 1989;23(12):810-4.

4. Jasper JJ, McNamara JA. The correction of interarch malocclusions using a fixed force module. Am J Orthod. 1995;108(6):641-50.

5. Hilgers JJ. The Pendulum appliance for class II noncompliance therapy. J Clin Orthod.1992;26(11):706-14.

6. Scuzzo G, F. Pisani, K. Takemoto and S. Della Vecchia. The Modified pendulum appliance with removable arms. J Clin Orthod. 2000;34:244-6.

7. Kircelli BH, Pektas ZO, Kircelli C. Maxillary molar Distalization with a Bone-anchored Pendulum Appliance. Angle Orthod. 2006;76(4):650-9.

8. Gianelly AA, Vaitas AS, Thomas WH, Berger DG. Distalization of molars with repelling magnets. A case report. J Clin Orthod. 1988; 22(1):40-44.

9. Bvloff F, Darendeliler MA, Stoff F. Mandibular molar distalizationwith the franzulum appliance. J Clin Orthod. 2000;34(9):518-32.

10. Jones RD, Whilt MJ. Rapid class II molar correction with open-coil jig. J Clin Orthod. 1992;26(10):661-4. 
11. Graber Vanarsdall Vig. Treatment options for sagittal corrections in Noncompliant Patients. $4^{\text {th }}$ edition, Elsevier Mosby publication. Chapter 21: 879-900.

12. Kalra V. The K-Loop molar distalizing appliance. J Clin Orthod. 1995; 29(5):298- 301.

13. Vogt W. The Forsus Fatigue Resistant Device. J Clin Orthod. 2006;40(6):368-377.

14. Jones G, Buschang PH, Kim KB, Oliver DR. Class II non-extraction patients treated with the Forsus fatigue resistant device versus intermaxillary elastics. Angle Orthod. 2008;78(2):332-338.

15. Greenfield RL. Fixed piston appliance for rapid class II correction. J Clin Orthod. 1995;29(3)0:174-83.

16. Carano, A. and Testa M .The Distal Jet for upper molar distalization. J Clin Orthod. 1996;30(7):374-380.

17. Gero S. M. Kinzinger GS, Gülden N, Faruk Yildizhan F, Diedrich PR. Efficiency of a skeletonized distal jet appliance supported by miniscrew anchorage for noncompliance maxillary molar distalization. Am J Orthod Dentofacial Orthop. 2009;136(4):578-86.

18. Locatelli R, Bednar J, Dietz VS, Gianelly AA. Molar distalization with superelastic NiTi wire. J Clin Orthod. 1992;26(5):277-9.

19. Sugawara $\mathbf{J}$ et al. Distal movement of mandibular molars in adult patients with the skeletal anchorage system. Am J Orthod Dentofacial Orthop. 2004;125(2):130-8.

20. De Clerck H, Geerinckx V, Siciliano S. The zygoma anchorage system. J Clin Orthod. 2002;36(8):455-9. 